\title{
ARRHYTHMIAS
}

\section{Searching for a simple way to STOP AF}

Cryoballoon ablation was developed to be a single-delivery technique for isolation of the pulmonary veins in patients with atrial fibrillation (AF). The STOP AF Cryoablation Investigators have published the results of their prospective, multicenter, randomized, controlled trial to assess the safety and efficacy of the cryoballoon ablation approach in patients with AF who are resistant to at least one antiarrhythmic drug.

Of the 245 patients with paroxysmal (78\%) or early persistent (22\%) AF, 163 were randomly assigned to undergo cryoballoon ablation and 82 were assigned to receive antiarrhythmic drugs. Mean procedure duration was $371 \mathrm{~min}$. After 12 months follow-up, freedom from chronic treatment failure was achieved in $69.9 \%$ of patients in the cryoablation group and in $7.3 \%$ of the antiarrhythmic drug group. Pulmonary vein stenosis occurred in $3.1 \%$ of cryoablated patients. Phrenic nerve paralysis was documented to have occurred in $11.2 \%$ of cryoballoon ablation procedures;
25 of the 28 patients who experienced phrenic nerve paralysis showed complete radiographic resolution over $144 \pm 27$ days.

In an editorial that accompanied the trial report, Dr Sabine Ernst (London, UK) points out that "when used by experienced electrophysiologist operators at the beginning of their learning curve with the device, a substantial number of serious complications were observed", and concludes that "cryoablation does not seem to be a contender to open the AF ablation field yet to nonelectrophysiologists". Dr Ernst believes that "the challenge of a truly simple ablation tool that will quickly and effectively isolate pulmonary veins has not yet been solved".

Bryony M. Mearns

Original article Packer, D. L. et al. Cryoballoon ablation of pulmonary veins for paroxysmal atrial fibrillation: first results of the North American arctic front (STOP AF) pivotal trial. J. Am. Coll. Cardiol. doi:10.1016/ j.jacc.2012.11.064 